\title{
Trajetória de Adolescentes em Conflito com a Lei Após Cumprimento de Medida Socioeducativa em Meio Fechado
}

\author{
Fernanda Ludke Nardi \\ Débora Dalbosco Dell’Aglio \\ Universidade Federal do Rio Grande do Sul \\ Porto Alegre, RS, Brasil
}

\begin{abstract}
RESUMO
Esta pesquisa investigou trajetórias de adolescentes após cumprimento de medida socioeducativa de internação, através de estudo quantitativo e longitudinal. Participaram 143 adolescentes em conflito com a lei com idades entre 14 e 20 anos. Foi aplicada uma ficha de dados sociodemográficos e um questionário sobre fatores de risco e de proteção, além da realização de contatos telefônicos por um período de dois anos e meio. Os participantes foram divididos em quatro grupos: G1: reincidentes ( $n=22), G 2$ : reinseridos socialmente $(n=24), G 3$ : manutenção da situação ( $n=38)$ e G4: outras situações $(n=59)$. Os resultados indicaram que as variáveis contextuais não apresentaram associação com o tipo de trajetória. Quanto às variáveis pessoais, observou-se diferenças significativas, sendo que G2 apresentou níveis mais elevados em relação à autoestima enquanto G1 apresentou níveis mais altos de uso de drogas. Portanto, propostas de intervenção junto a essa população devem considerar tanto variáveis contextuais como variáveis pessoais.
\end{abstract}

Palavras-chave: Adolescente em conflito com a lei; Autoestima; Drogas.

\begin{abstract}
Path of Young Offenders After a Socio-Educational Measure Deprived of Freedom

This research investigated trajectories of adolescents after a socio-educational measure through a quantitative and longitudinal study. Participants were 143 young offenders aged between 14 and 20 years old. The instruments used were a data socio-demographic and a risk and protective factors questionnaire, besides conducting telephone contacts for a period of two and half years. Participants were divided into four groups: G1: recidivists ( $\mathrm{n}=22)$, G2: socially reintegrated $(n=24), G 3$ : maintaining the status $(n=38)$ and $G 4$ : other situations $(n=59)$. The results indicated that the contextual variables were not associated with the type of trajectory. As to personal variables, we found significant differences, G2 presented higher levels in relation to self-esteem while G1 had higher levels of drug use. Therefore, proposals for intervention with this population should consider both contextual and personal variables.
\end{abstract}

Keywords: Teenager in conflict with the law; Self-esteem; Drugs.

\section{RESUMEN}

Trayectorias de Adolescentes en Conflicto con la Ley Después de Cumplimiento de Medida Socioeducativa de Internación

Este estudio investigo trayectorias de adolescentes después de cumplimiento de medida socioeducativa de internación, a través de una investigación cuantitativa y longitudinal. Participaron 143 adolescentes en conflicto con la ley, con edades entre 14 y 20 años, que llenaron un formulario de datos sociodemográficos y un cuestionario sobre factores de riesgo y de protección. A partir de esos instrumentos, se mantuvo contacto telefónico por un periodo de dos años y medio. Los participantes fueron divididos en cuatro grupos: G1: reincidentes $(n=22)$; $G 2$ : reinsertados $(n=24)$; G3: misma situación $(n=38)$; y G4: otras situaciones $(n=59)$. Los resultados indicaron que las variables contextuales no presentan asociación con el tipo de trayectoria. Cuanto a las variables personales, se observó diferencias significativas, pues G2 presentó niveles más elevados de autoestima mientras G1 presentó niveles más altos de consumo de drogas. Se concluye que propuestas de intervención con estos adolescentes deben considerar tanto variables contextuales como variables personales.

Palabras clave: Adolescentes en conflicto con la ley; Autoestima; Drogas. 
A trajetória de adolescentes em conflito com a lei tem despertado interesse tanto na população em geral como na comunidade científica, tendo em vista o impacto causado sobre o desenvolvimento dos próprios adolescentes assim como sobre a sociedade. Essas trajetórias estão permeadas por uma variedade de objetivos e funções, as quais se modificam ao longo do desenvolvimento. Torna-se importante, assim, considerar quais fatores influenciam os caminhos desses jovens, avaliando a existência ou não de mudança em seus comportamentos ao longo do tempo (Iselin, Mulvey, Loughran, Chung, \& Schubert, 2012; Silva, 2002). Pacheco, Alvarenga, Reppold, Piccinini e Hutz (2005) destacam que, havendo a manutenção das características do ambiente social do indivíduo, os comportamentos antissociais tendem a persistir e muitas vezes a se agravar ao longo do desenvolvimento.

De acordo com Hein (2004), o comportamento infracional juvenil pode se desenvolver de duas formas: de forma esporádica e de forma persistente. Moffitt, em 1993, já apontava nessa mesma direção quando propôs um modelo clássico para a caracterização da heterogeneidade dos comportamentos antissociais. Conforme a autora, podem ser identificados dois tipos de comportamento antissocial, os quais se diferenciam de acordo com a idade de início. Um deles estaria restrito a um pequeno grupo de indivíduos, tendo origem no desenvolvimento neurológico e manifestando-se desde a infância até a idade adulta. Fatores de risco individuais da criança, tais como déficit cognitivo e hiperatividade, se associariam a fatores ambientais, como práticas educativas inadequadas e pobreza, resultando em um comportamento antissocial persistente e severo. O outro tipo estaria relacionado a um grupo maior de indivíduos que iniciam comportamentos antissociais em meados da adolescência, mas desistem de tal conduta no início da idade adulta.

Segundo Hein (2004), durante a adolescência, os jovens exploram e experimentam diversos comportamentos, inclusive antissociais, os quais seriam vistos como comuns e normativos. A mudança no comportamento antissocial no início da idade adulta estaria relacionada a aspectos da personalidade e questões biológicas que mudam com a idade, tais como a produção de neurotransmissores associados ao comportamento antissocial (por exemplo, a dopamina e noradrenalina acetilcolina) que diminue ao longo do curso da vida (Collins, 2004). O estudo de Lee, Klaver, Hart, Moretti e Douglas (2009), com uma amostra de 83 adolescentes em conflito com a lei do sexo masculino, de centros de detenção juvenil no Canadá, revelou que de fato a maioria dos participantes apresentou uma diminuição da pontuação, ao longo do tempo, no instrumento que media traços de psicopatia, o Psychopathy Checklist: Youth Version (PCL:YV).

Outras pesquisas também têm enfatizado a questão da trajetória de adolescentes com comportamentos antissociais. A partir de uma amostra de 566 adolescentes americanos em situação de vulnerabilidade social, foi conduzido um estudo longitudinal entre os anos de 1998 e 2005, identificando três trajetórias: um grupo com baixo risco, um grupo com alto risco e outro grupo também com alto risco, no qual se observou uma queda dos comportamentos antissociais após os 16 anos de idade. $\mathrm{O}$ segundo grupo apresentou chance dez vezes maior de ser preso quando comparado ao grupo 1, além de constituir-se no grupo com maior risco para o envolvimento em consumo de álcool e outras drogas. De acordo com os autores, embora seja difícil avaliar a sequência entre uso de substâncias e comportamento antissocial, é provável que o envolvimento com drogas afete a trajetória de comportamento antissocial (Park, Lee, Sun, Vazsonyi, \& Bolland, 2010). O estudo de Nijhof et al. (2011) também revelou a existência de três grupos de jovens com comportamento antissocial: grupos com escores baixos, moderados e altos no instrumento Youth Psychopathic Traits Inventory (YPI), sendo que os dois últimos apresentaram escores elevados para o uso de drogas. De modo geral, Moffitt (2006) menciona que a maioria das pesquisas na área da delinquência juvenil confirma que o início de comportamentos antissociais na infância prediz mau ajustamento a longo prazo. Conforme a autora, os dois primeiros tipos de comportamentos antissociais - (1) que persistem até a adultez e (2) que cessam no final da adolescência e início da idade adulta - englobam a maioria da população de jovens infratores.

Assim, diversas pesquisas têm tentado investigar os fatores que levariam a seguir uma ou outra trajetória de comportamento antissocial. Alguns estudos revelaram que a estabilidade no emprego e o apego conjugal na adultez contribuem para mudanças significativas na desistência de comportamentos criminosos (Horney, Osgood, \& Marshall, 1995; Warr, 1998). A pesquisa de Mennis e Harris (2011), baseada em um banco de dados de 7166 meninos infratores americanos, revelou um percentual de reincidência de $40 \%$, dos quais a maioria dos atos infracionais estava relacionada às drogas, como posse ou tráfico.

Domburgh et al. (2009) encontraram, em seu estudo, que jovens que desistiram da trajetória delitiva estavam mais envolvidos em atividades familiares e vivendo em famílias menores, do que jovens que persistiram com comportamentos antissociais. Mennis e Harris (2011) mencionaram ainda a influência dos pares, a qual atuaria como facilitadora da repetição do ato infracional. Outro estudo (Maldonado-Molina, 
Piquero, Jennings, Bird, \& Canino, 2009) mostrou diferenças significativas entre diversos fatores de risco e de proteção através das trajetórias dos adolescentes infratores, tais como gênero, autoestima, exposição à violência e eventos de vida estressantes. Nesse estudo, os jovens do grupo que desistiram da trajetória delitiva exibiram níveis mais altos de autoestima quando comparados ao grupo com trajetória delinquente.

Alguns autores propõem diferentes teorias sobre aspectos que reforçam ou não a continuidade dos comportamentos antissociais ao longo do desenvolvimento. De acordo com Higgins et al. (2011), os indivíduos que continuam a cometer delitos, o fazem porque são reforçados de diferentes maneiras. $\mathrm{O}$ reforço pode ocorrer de duas formas - social e não social. No reforço social, outros indivíduos, como amigos e familiares, contribuiriam de maneira positiva ou negativa para o comportamento do adolescente. No reforço não social, aspectos psicológicos e biológicos do próprio indivíduo atuariam como reforço, sendo que este tipo estaria mais relacionado ao uso de drogas, ao crime e à delinquência. Um estudo realizado com 504 jovens de Tocantins, no Brasil, revelou características individuais que atuariam como reforço não social, como a busca de intensidade e de novidades. Esses resultados, segundo os autores, permitem supor que as variáveis de personalidade têm bastante contribuição quando se pretende explicar a conduta antissocial (Formiga, Aguiar, \& Omar, 2008). No estudo de Higgins et al. (2011), os adolescentes com comportamento antissocial limitado à adolescência mostraram-se menos suscetíveis ao reforço não social.

Agnew e White (1992) propõem a "General Strain Theory" (GST), que relaciona alguns comportamentos do indivíduo, como a delinquência, com a tensão. O termo tensão é definido pelos autores como relações nas quais o indivíduo não é tratado como gostaria de ser. Porém, posteriormente o termo foi relacionado também a eventos e condições em que o indivíduo sente-se desconfortável. A exposição a essas situações intensificaria emoções negativas como raiva e frustração, criando constante tensão sobre o indivíduo. De acordo com essa teoria, as falhas são atribuídas principalmente ao indivíduo (Agnew, 2001), desenvolvendo pobre autoconceito e desesperança, características fortemente associadas ao comportamento antissocial nos adolescentes (Park et al., 2010). Iselin et al. (2012) discorrem a respeito dessa teoria relacionando-a com a desistência do comportamento antissocial. Segundo os autores, quando as pessoas não conseguem atingir as metas positivamente valorizadas, elas podem se engajar em comportamentos criminosos como forma de lidar com a tensão de seus desejos não satisfeitos. Assim, possuir identidades positivas a respeito de si mesmo e acreditar que as metas podem ser alcançadas através de atividades lícitas, auxilia na desistência do comportamento antissocial.

Diante das considerações teóricas a respeito do desenvolvimento do comportamento antissocial, pode-se pensar nas diferenças existentes entre os jovens que seguem a trajetória delitiva e os que não seguem. Assim, o objetivo do presente estudo foi analisar as diferentes trajetórias de adolescentes que cumpriram medida socioeducativa de internação, através de um estudo longitudinal. Como objetivos específicos, foram observados os fatores de risco e de proteção presentes nas trajetórias dos adolescentes que reincidiram e dos que foram reinseridos socialmente, a partir de variáveis pessoais e contextuais.

\section{MÉTODO}

\section{Delineamento e contextualização}

Foi desenvolvido um estudo quantitativo, longitudinal, com a realização de duas coletas de dados (T1 e T2) com intervalo mínimo de um ano, incluindo aplicação de instrumentos e contatos telefônicos. Essa pesquisa faz parte de um estudo maior intitulado "Adolescência em Diferentes Contextos: Família e Institucionalização", o qual tem como objetivo geral avaliar longitudinalmente características pessoais e familiares de adolescentes em diferentes contextos, observando a presença de fatores de risco e de proteção. Este estudo abrange a população específica de adolescentes em conflito com a lei que cumpriam medida em regime fechado na Fundação de Atendimento Socioeducativo (FASE-RS), a qual possui cinco unidades de internação, sendo uma feminina.

\section{Participantes}

Participaram da pesquisa 143 adolescentes em conflito com a lei que cumpriam medida de internação na FASE. Os participantes tinham entre 14 e 20 anos $(\mathrm{M}=17,23, \mathrm{dp}=1,19)$, sendo que $128(89,5 \%)$ eram do sexo masculino e $15(10,5 \%)$ do sexo feminino. Segundo a Assessoria de Informação e Gestão da FASE-RS, no período da coleta de dados, havia 565 meninos internados na instituição e 31 meninas, sendo que a amostra representou respectivamente $23 \%$ dos meninos e $48 \%$ das meninas em cumprimento de medida em regime fechado. A maioria dos adolescentes estava entre a $5^{\mathrm{a}}$ e $6^{\mathrm{a}}$ séries e $60 \%$ deles era de cor branca, enquanto $26,6 \%$ de cor negra.

\section{Instrumentos}

Na primeira etapa da coleta de dados, os participantes responderam a uma ficha de dados referente às visitas 
de familiares, ao ato infracional cometido, entre outros, e a uma versão adaptada do Questionário de Juventude Brasileira Versão II (Dell'Aglio, Koller, CerqueiraSantos, \& Colaço, 2011). Esse questionário foi reduzido para a coleta de dados na FASE, sendo composto por 47 questões objetivas sobre fatores de risco e de proteção, incluindo questões sobre expectativas de futuro, eventos estressores e a Escala de Autoestima de Rosenberg (Rosenberg, 1989). Na segunda etapa da coleta de dados, um novo questionário foi aplicado nos adolescentes que ainda permaneciam na FASE, contendo as mesmas questões, porém adaptadas para abarcar somente os acontecimentos dos últimos 12 meses.

\section{Procedimentos e considerações éticas}

O projeto foi aprovado pelo Comitê de Ética em Pesquisa do Instituto de Psicologia da UFRGS (protocolo $n^{\circ}$ 2009060). A FASE autorizou a realização da pesquisa e foram coletados dados nas cinco unidades de internação. Os adolescentes que tinham escolaridade mínima de $5^{\mathrm{a}}$ série do Ensino Fundamental (critério utilizado para garantir mais condições de compreensão dos instrumentos) foram convidados a participar. No entanto, alguns adolescentes com menor escolaridade, mas que desejaram participar, também responderam individualmente ao questionário com o auxílio dos pesquisadores. Os adolescentes assinaram o Termo de Consentimento Livre e Esclarecido e a aplicação dos instrumentos foi coletiva, em grupos de 6 a 8 jovens, com duração aproximada de 60 minutos. Aqueles que desejavam participar da segunda etapa da coleta de dados forneceram informações sobre seus contatos (números de telefone).

Os participantes foram acessados ao longo de dois anos e meio. Na primeira coleta de dados foi feita aplicação dos instrumentos durante os meses de março a outubro de 2010. A segunda coleta englobou uma nova aplicação dos instrumentos durante os meses de março a outubro de 2011, com adolescentes que permaneciam na FASE. Para acessar os que já haviam saído da instituição, foram realizados contatos telefônicos com os próprios adolescentes ou familiares, com o objetivo de investigar a situação atual dos mesmos após a internação. Essas ligações foram feitas no mês de junho de 2011 e nos meses de janeiro e junho de 2012. Além desses procedimentos, foi feito contato com uma assistente social do Presídio Central de Porto Alegre (Superintendência dos Serviços Penitenciários do Estado do Rio Grande do Sul SUSEPE) e com psicólogas e assistentes sociais das instituições Calábria e Pão dos Pobres, onde é efetuado o Programa de Oportunidades e Direitos (POD). Esse Programa foi instituído através do projeto de Lei $n^{0} 295 / 2008$, com a finalidade de auxiliar a inserção familiar, educacional e profissional do adolescente e do jovem adulto egressos da FASE. Foi enviada uma lista com o nome dos adolescentes participantes da primeira etapa da coleta para os três locais, com o objetivo de averiguar informações sobre suas trajetórias. Também foram realizados contatos com os Centros de Referência Especializados de Assistência Social (CREAS), locais responsáveis pela execução de medidas socioeducativas em meio aberto. Porém, apenas três adolescentes estavam cumprindo medida em meio aberto após a medida de internação, sendo que um deles foi para o Presídio Central de Porto Alegre após novo ato infracional.

\section{Análise de dados}

Foram realizadas análises descritivas observando as trajetórias dos adolescentes e classificando-os em grupos. Para avaliar diferenças entre os grupos foram utilizados os testes não paramétricos Qui-quadrado e Mann Whitney, com as seguintes questões do questionário:

Comportamento antissocial. Foi investigado através de sete itens, respondidos de forma dicotômica $(0=N a ̃ o, 1=S i m)$ : "Envolvimento em brigas com agressão física/violência contra pessoas", "Destruição de propriedade", "Envolvimento em pichação", "Assaltou alguém", "Roubou algo", "Vendeu drogas" e "Outras" (e.g., porte de arma, homicídio e latrocínio). O escore total foi computado somando-se o número de atividades ilícitas em que o jovem se envolveu.

Violência intra e extrafamiliar. Foram investigadas através dos seguintes itens, respondidos de forma dicotômica $(0=N a ̃ o, 1=\operatorname{Sim})$ : "ameaça ou humilhação", "soco ou surra", "agressão com objeto", "mexeu no meu corpo contra minha vontade" e "relação sexual forçada". O escore total foi computado somando-se o número de respostas positivas.

Expectativas de futuro. A questão sobre as expectativas para o futuro foi baseada no instrumento construído por Günther e Günther (1998), contendo nove itens em escala tipo Likert com cinco opções de resposta que avaliavam quais as chances que acreditavam ter de terminar o ensino médio, ingressar em uma universidade, ter um emprego, ter uma família, entre outros $(1=$ Chances muito baixas, $5=$ Chances muito altas). $\mathrm{O}$ escore total foi computado através da soma dos escores dos itens.

Uso de drogas. As substâncias investigadas foram álcool, cigarro, cola ou solventes, maconha, cocaína, crack e ecstasy. A pergunta referia-se ao uso no período antes de ingressar na instituição. Os itens foram dispostos no formato dicotômico $(0=$ Não, $1=\operatorname{Sim})$ e 
o escore total foi computado somando-se o número de respostas positivas.

Percepção acerca do relacionamento familiar: Foi investigada através de 15 itens, tais como "meus pais raramente me criticam" e "eu me sinto aceito pelos meus pais", avaliados em escala Likert de cinco pontos $(1=$ Discordo totalmente, $5=$ Concordo totalmente $) . \mathrm{O}$ escore total foi computado através da soma dos escores dos itens.

Eventos estressores: Foi avaliada a ocorrência de vinte eventos estressores, tais como "alguém em minha casa está desempregado", "já morei na rua", "alguém da minha família está ou esteve preso" e "alguém muito importante pra mim faleceu". Os itens foram dispostos no formato dicotômico $(0=N a \tilde{o}, 1=S i m)$ e o escore total foi computado somando-se o número de respostas positivas.

Autoestima: Foi avaliada através da Escala de Autoestima de Rosenberg - EAR (Rosenberg, 1989), a qual teve suas propriedades psicométricas atualizadas no Brasil por Hutz e Zanon (2011). Os itens investigaram aspectos positivos (ex. "Eu acho que tenho muitas boas qualidades") e negativos (Ex.: "Levando tudo em conta, eu me sinto um fracasso") do autovalor. As opções de resposta variaram entre 1 (Nunca) e 5 (Sempre).

\section{RESULTADOS}

Conforme a Tabela 1, dos 143 adolescentes que participaram do estudo na primeira etapa, 38 perma- neciam internados na FASE e 105 já haviam saído da instituição até o final da coleta de dados, seguindo diferentes trajetórias. Os participantes foram divididos em quatro grupos: Grupo 1 - Reincidentes - aqueles que haviam retornado para o sistema socioeducativo (FASE) ou estavam inseridos no sistema prisional (Presídio Central de Porto Alegre-SUSEPE); Grupo 2 Reinseridos socialmente - englobou nove adolescentes que estavam inseridos em cursos profissionalizantes (POD) e 15 que estavam estudando e/ou trabalhando; Grupo 3 - Manutenção da situação - aqueles que continuavam cumprindo medida socioeducativa de internação na FASE; Grupo 4 - Outras situações englobou aqueles jovens que haviam falecido $(n=2)$ e que estavam com problemas de saúde $(\mathrm{n}=3)$, tais como internação decorrida de dependência química, ou que não foi possível realizar contato $(n=54)$.

Foram realizadas análises observando as relações entre variáveis pessoais e contextuais e as diferentes trajetórias dos adolescentes (Grupo 1 - reincidentes versus Grupo 2 - reinseridos socialmente). Em relação às variáveis contextuais, foram observadas as frequências e percentuais por grupo nas seguintes variáveis: morar com o pai, morar com a mãe, ter familiar que usa drogas, ter sido reprovado, ter sido expulso da escola, exposição à violência intra e extrafamiliar e ocorrência de eventos estressores. As análises, através do teste qui-quadrado e do teste Mann-Whitney, não indicaram associações significativas dessas variáveis contextuais com os grupos 1 e 2 (Tabela 2).

TABELA 1

Características dos participantes por grupo

\begin{tabular}{|c|c|c|c|c|c|c|}
\hline Grupo & Média idade & $D P$ & Masculino & Feminino & Total & $\%$ \\
\hline G1 - Reincidentes & 17,55 & 0,97 & 21 & 01 & 22 & 15 \\
\hline G2 - Reinseridos socialmente & 17,67 & 0,91 & 22 & 02 & 24 & 17 \\
\hline G3 - Manutenção da situação & 16,71 & 1,03 & 33 & 05 & 38 & 27 \\
\hline G4 - Outras situações & 17,28 & 1,34 & 52 & 07 & 59 & 41 \\
\hline
\end{tabular}

TABELA 2

Resultados das variáveis contextuais por grupo

\begin{tabular}{lcccc}
\hline \multicolumn{1}{c}{ Variável } & $\begin{array}{c}\text { G1- Reincidentes } \\
\%\end{array}$ & G2-Reinseridos socialmente & $\chi^{2}$ & $p$ \\
\hline Morava com pai & 40,9 & 33,3 & 0,05 & 0,82 \\
Morava com mãe & 72,7 & 66,7 & 0,01 & 0,90 \\
Ter familiar que usa drogas & 50 & 47,8 & 0,00 & 1 \\
Foi reprovado & 100 & 91,7 & 0,43 & 0,51 \\
Foi expulso & 40 & 37,5 & 0,00 & 1 \\
& Mean Rank & Mean Rank & Mann-Whitney & $p$ \\
Exposição à violência intra & 21,25 & 23,75 & 214,50 & 0,49 \\
Exposição à violência extra & 21,68 & 22,33 & 224,00 & 0,86 \\
Eventos estressores & 16,81 & 19,00 & 133,00 & 0,54 \\
\hline
\end{tabular}


TABELA 3

Resultados das variáveis pessoais por grupo

\begin{tabular}{|c|c|c|c|c|}
\hline \multirow{2}{*}{ Variável } & G1-Reincidentes & G2-Reinseridos socialmente & \multirow{2}{*}{ Mann-Whitney } & \multirow{2}{*}{$p$} \\
\hline & Mean Rank & Mean Rank & & \\
\hline Autoestima & 17,00 & 25,20 & 126,00 & 0,02 \\
\hline Uso de drogas & 20,71 & 13,06 & 73,00 & 0,02 \\
\hline Comportamento antissocial & 24,30 & 18,43 & 158,50 & 0,11 \\
\hline Expectativas de futuro & 19,78 & 22,17 & 185,50 & 0,52 \\
\hline
\end{tabular}

Em relação às variáveis pessoais, foram observadas autoestima, uso de drogas, comportamento antissocial e expectativas de futuro. De acordo com a Tabela 3, acima, pode-se observar que houve diferença significativa entre os dois grupos quanto às variáveis autoestima e uso de drogas. O G2 apresentou níveis mais elevados em relação à autoestima enquanto o G1 apresentou níveis mais altos de uso de drogas. Embora a média de manifestação de comportamentos antissociais tenha sido maior no G1 e a média de expectativas futuras tenha sido mais alta em G2, estas variáveis não apresentaram diferença significativa.

\section{DISCUSSÃO}

Os resultados deste estudo indicaram que 24 participantes $(17 \%)$ apresentaram trajetórias positivas após a internação na FASE, sendo elas trabalho, estudo ou inserção em curso profissionalizante. Diversas pesquisas realizadas no contexto brasileiro (Assis \& Souza, 1999; Gallo \& Williams, 2008; Silva, 2002) retratam o trabalho e o estudo como fatores de proteção importantes para o desenvolvimento dos jovens, na medida em que auxiliam na concretização de projetos para o futuro. Em contrapartida, 22 adolescentes parecem ter se mantido numa trajetória delitiva, visto que retornaram para o sistema socioeducativo ou prisional. O estudo de Silva (2002), com 200 adolescentes infratores do sexo masculino, revelou que 53 jovens $(26,5 \%)$ permaneceram cometendo delitos e $145(72,5 \%)$ finalizaram o cumprimento da medida, não ingressando mais no sistema socioeducativo após dois anos e meio, a partir de informações da justiça.

Ressalta-se, no entanto, que não foi possível obter dados a respeito da trajetória de 54 adolescentes, devido à perda de contato com os mesmos. Esse aspecto gerou uma reflexão a respeito da fragilidade do acompanhamento a esses jovens após o cumprimento de medida socioeducativa. De acordo com o Sistema Nacional de Atendimento Socioeducativo (SINASE), instituído nacionalmente através da Lei 12.594, de 18 de janeiro de 2012, apenas há previsão de acompanhamento para os casos de adolescentes em cumprimento de medida socioeducativa que apresentem indícios de transtorno mental, de deficiência mental, ou associadas. Em outras situações, é realizado um Plano Individual de Atendimento (PIA) para cada jovem antes de sua saída do regime de internação. Porém, após a extinção da medida, não é feito nenhum acompanhamento por profissionais a fim de verificar se o PIA está sendo efetivado pelo jovem e sua família. No entanto, algumas situações podem ser caracterizadas como formas de acompanhamento ao jovem após a medida de meio fechado, como, por exemplo, (a) quando o adolescente recebe alguma medida em meio aberto, como liberdade assistida ou prestação de serviços à comunidade; ou (b) quando o adolescente ingressa voluntariamente no POD, onde recebe acompanhamento pelo prazo de um ano ou mais, caso continue inserido em algum curso profissionalizante. Dessa forma, neste estudo, houve dificuldade para acessar os jovens após a medida de internação, visto que poucos participantes ingressaram no POD e/ou receberam medidas em meio aberto. Portanto, a maioria dos adolescentes egressos da FASE não recebeu acompanhamento de profissionais no seu retorno à comunidade, tornando-se mais difícil sua localização.

Podem, ainda, ser consideradas outras hipóteses quanto à impossibilidade de acessar esses participantes. De acordo com o Mapa da Violência (2013), a taxa total de mortalidade da população brasileira caiu, enquanto que a taxa de mortalidade juvenil manteve-se praticamente estagnada, ainda com um leve aumento. As epidemias e doenças infecciosas, que eram as principais causas de morte entre os jovens cinco ou seis décadas atrás, foram sendo progressivamente substituídas pelas denominadas causas externas, principalmente acidentes de trânsito e homicídios. Neste estudo, foram constatados dois homicídios entre os participantes ao longo do período de coletas de dados. Porém, pode-se considerar que o número de homicídios poderia ser maior, caso tivesse sido possível acessar informações sobre todos participantes. Ainda conforme o Mapa (2013), apesar de os jovens 
representarem aproximadamente $18 \%$ da população total, o número de assassinatos nessa faixa gira em torno de $36 \%$. Tendo em vista que o contato com a criminalidade, realidade de todos os participantes deste estudo, pode aumentar o percentual de homicídio, acredita-se que mais adolescentes podem ter sido vítimas de violência durante a realização da pesquisa.

A partir da classificação dos participantes nos grupos identificados, foram analisadas suas características. A trajetória do G2 pode sugerir que esses adolescentes possuíam comportamentos antissociais limitados à fase da adolescência, tendo em vista sua inserção positiva após o desligamento da FASE, o que tem sido descrito por Park et al. (2010) como grupo de baixo risco. Em contrapartida, os participantes do G1 persistiram com a conduta antissocial, podendo encaixar-se no grupo que Moffitt (1993) nomeou como comportamento antissocial persistente ou de alto risco. A classificação nos grupos, no entanto, não é determinante, podendo oscilar ao longo do desenvolvimento, já que o processo se mostra muito dinâmico e influenciado por diversas variáveis pessoais e contextuais. Dessa forma, adolescentes que retornaram para a FASE ou jovens que ingressaram no presídio poderão, no futuro, apresentar trajetórias positivas, de inserção social, enquanto outros que, atualmente, foram reinseridos socialmente poderão ainda apresentar reincidência no comportamento infracional. De qualquer forma, apenas estudos longitudinais, que acompanhem os egressos por períodos mais longos, permitirão uma análise mais aprofundada sobre as trajetórias desses jovens.

Também foi possível analisar características pessoais e contextuais dos grupos identificados, observando fatores de risco e de proteção. Os resultados indicaram que as variáveis contextuais não contribuíram, nesse estudo, para o seguimento de uma ou outra trajetória, visto que não houve diferença entre os grupos. Foi observado que os dois grupos estavam expostos de forma similar às variáveis contextuais não viver com o pai ou a mãe, ter familiar que usa drogas, ter sido reprovado, ter sido expulso da escola, exposição à violência intra e extrafamiliar e ocorrência de eventos estressores, que em geral representam riscos ao desenvolvimento. No estudo de Domburgh et al. (2009), embora mais adolescentes persistentes nas atividades delitivas tenham sido expostos a fatores de risco, a diferença entre os grupos também não foi significativa.

Entretanto, as variáveis pessoais autoestima e uso de drogas apresentaram diferenças significativas entre os grupos, indicando a presença de associação à manifestação do comportamento infrator. Donnellan, Trzesniewski, Robins, Moffitt e Caspi (2005), em estudo sobre a relação entre autoestima e comportamentos externalizantes, tais como o antissocial, apresentaram diferentes pontos de vista. Por um lado, os autores apontam uma relação entre baixa autoestima e comportamento antissocial, por outro, assinalam possível associação entre altos níveis de autoestima e atividades criminosas. Entretanto, essa última estaria mais relacionada ao construto de narcisismo. Estudo brasileiro com adolescentes autores de ato infracional (Silva, 2002) revelou bons níveis de autoestima nos participantes, sugerindo associação entre autoestima e delinquência juvenil. Porém, os dados deste estudo, ao se comparar G1 e G2, indicaram que níveis mais altos de autoestima estão significativamente associados à desistência de comportamentos antissociais.

De acordo com Trzesniewski, Donnellan e Robins (2003), a autoestima é considerada um dos principais fatores associados a resultados positivos na adolescência e na vida adulta. Além disso, a autoestima relaciona-se com o desenvolvimento do auto-conceito. Conforme a "General Strain Theory" (GST), proposta por Agnew e White (1992), exibir bons níveis nessas variáveis contribui para a forma como o adolescente irá enfrentar as situações de tensão. De forma oposta, sentimentos de inferioridade e insegurança, surgidos muitas vezes de situações de tensão, motivariam comportamentos antissociais (Donnellan et al., 2005). Assim, pode-se considerar a hipótese de que bons níveis de autoestima permitem ao jovem ter mais confiança em si mesmo, possuindo mais segurança para efetuar mudanças em sua trajetória delitiva e buscando alternativas mais positivas de desenvolvimento. No entanto, cabe destacar que não há consenso sobre o efeito da variável autoestima sobre o comportamento antissocial, sendo necessários mais estudos para elucidar essas associações.

Quanto ao uso de drogas, o grupo dos jovens que continuaram a cometer delitos após a internação na FASE mostrou níveis mais altos quando comparado ao grupo que desistiu dos comportamentos antissociais. Outros estudos que investigaram trajetórias de jovens infratores encontraram associação entre o uso de drogas e o comportamento delitivo (Mason \& Windle, 2002; Nijhof et al., 2011; Park et al., 2010). No Brasil, Pereira e Sudbrack (2008), assim como Zappe e Dias (2012), também apontam várias conexões entre os níveis de dependência relacional das drogas (efeitos, crenças, relações afetivas, pares, provedores, fornecedores) e a prática de infrações.

De acordo com Horney et al. (1995), os indivíduos estão mais propensos a cometer crimes quando estão sob o efeito de drogas. Wainer (2006) corrobora essa ideia mencionando que a relação entre o consumo de drogas 
e a infração juvenil é preocupante porque há uma maior probabilidade de que jovens usuários de drogas se envolvam em um novo delito e, ao reincidir, enfrentem consequências mais graves. Conforme Horney et al. (1995), a relação entre essas duas variáveis é espúria e não causal, ou seja, um fator acaba por estimular a ocorrência do outro formando um ciclo incessante. Essa relação levaria o adolescente a envolver-se cada vez mais no mundo do crime e das drogas (Wainer, 2006). A partir dos resultados deste estudo, pode-se observar que, além de estar relacionado à delinquência juvenil, o uso de drogas pode favorecer a manutenção da trajetória delitiva.

Assim, verifica-se que, embora as variáveis contextuais sejam importantes no desenvolvimento e manutenção de comportamentos antissociais, o que já vem sendo tratado por muitos estudos da área (Apel \& Burrow, 2011; Assis et al., 2009; Paton et al., 2009), as variáveis pessoais parecem apresentar um papel significativo na modificação dessas trajetórias.

\section{CONSIDERAÇÕES FINAIS}

O objetivo deste estudo foi analisar as diferentes trajetórias encontradas em uma amostra de adolescentes autores de ato infracional. Os resultados demonstraram que 24 participantes (17\%) alcançaram trajetórias positivas após a internação na FASE, envolvendo trabalho, estudo ou inserção em curso profissionalizante. Em contrapartida, 22 (15\%) adolescentes retornaram para o sistema socioeducativo ou prisional, devido à reincidência. Quanto às variáveis investigadas, observou-se diferença significativa entre os grupos quanto a duas variáveis pessoais: autoestima e uso de drogas. O grupo de adolescentes que estava trabalhando ou estudando apresentou níveis mais elevados em relação à autoestima e níveis mais baixos de uso de drogas. Destaca-se, no entanto, que não foi possível obter dados a respeito da trajetória de 54 participantes, devido à perda de contato com os mesmos, evidenciando a fragilidade do acompanhamento dos jovens ao saírem de um regime de internação. Dessa forma, pode-se questionar quais as chances que jovens em conflito com a lei, expostos a tantos fatores de risco e eventos estressores, têm para modificar suas trajetórias de vida sem um acompanhamento eficaz? Após um período de internação, os adolescentes retornam para suas comunidades, onde muitas vezes foi cometido o delito e onde há contato muito próximo com o crime e gangues, sem nenhum auxílio para enfrentar tudo isso. Além disso, a família também fica desamparada no momento em que se encerra o período de internação. Sem o devido acompanhamento e estrutura para a família e o jovem, torna-se mais difícil impedir que a trajetória delitiva permaneça em suas vidas.

Algumas limitações do presente estudo devem ser apontadas. Uma das limitações foi referente ao pequeno número de adolescentes acessados na segunda coleta de dados, tendo em vista a dificuldade em localizar participantes que já não se encontravam mais em medida fechada. Análises mais robustas poderiam ser utilizadas com uma amostra maior em T2. Assim, para suprir essa limitação, novos estudos devem contar com amostras maiores desde a primeira coleta de dados, considerando que sempre existem perdas em estudos longitudinais (Lee et al., 2009), especialmente junto à população estudada. Além disso, um número maior de instituições poderia ser acessado para obter dados sobre os adolescentes egressos, tais como unidades de saúde e de assistência social (Centros de Referência de Assistência Social). Diante da realidade de homicídios contra a juventude, o contato com o Instituto Médico Legal (IML) também poderia ser uma alternativa na busca por esses adolescentes.

Embora a realidade do sistema socioeducativo apresente um número bastante superior de meninos cumprindo medida em comparação às meninas (ANCED/Fórum DCA, 2004), outra limitação desse estudo foi a pequena quantidade de meninas, impossibilitando a avaliação de trajetórias de acordo com o sexo. Esse estudo teve como limitação, também, o fato de ter acessado somente as percepções dos próprios adolescentes em conflito com a lei. Investigar a percepção de outras fontes, tais como familiares e profissionais, permitiria uma triangulação dos dados, conferindo ao estudo maior validade ecológica.

Ressalta-se, a partir dos resultados encontrados, a importância das variáveis pessoais no que concerne à desistência do comportamento antissocial, sendo necessário que projetos de intervenção, assim como políticas públicas direcionadas a esta população possam contemplar esses aspectos. Abrams e Snyder (2010) mencionam a relevância de uma abordagem ecológica, por compreender todos os contextos em que se desenvolvem os jovens, incluindo aspectos como a família, a comunidade e a cultura. As teorias que orientam as intervenções atuais baseiam-se principalmente em uma abordagem individual e em intervenções familiares. A abordagem individual mostra-se importante principalmente por tratar de questões cruciais como a dependência química, além de outros transtornos mentais e de características de personalidade (Abrams \& Snyder, 2010). Moffitt (2006), baseada em seu modelo, menciona que as intervenções para aqueles jovens com características persistentes deveriam ocorrer desde a infância, pois 
envolveriam fatores como os apontados por este estudo, como a autoestima dos adolescentes. Portanto, propostas de intervenção junto a essa população devem considerar tanto variáveis contextuais como variáveis pessoais.

Assim, observa-se a necessidade de estudos que possibilitem um monitoramento das trajetórias destes jovens, de forma que se possa obter estatísticas mais verídicas, sendo possível avaliar a eficácia dos atendimentos e das políticas públicas destinadas a essa população, além da necessidade de programas de acompanhamento ao jovem, quando do retorno para a comunidade. Programas de ressocialização com características diversificadas para essa população devem ser pensados, visto que, dos 46 adolescentes egressos acessados na segunda etapa da coleta, apenas nove estavam inseridos no POD. Talvez outros programas de acompanhamento aos egressos possam ser idealizados para que um número maior de jovens possa ser beneficiado. Zapata (2010) ressalta que é importante perceber que muitos adolescentes tiveram socialização insuficiente dentro do núcleo familiar, dificultando que, logo de início, enfrentem com êxito a tarefa que lhes é imposta, a de aprendizado. Por isso, é necessário um trabalho pedagógico diferenciado. Além disso, a adesão ao POD, programa de acompanhamento aos egressos, é voluntária e a escolha é feita pelo próprio adolescente. Porém, ressalta-se que muitos deles, ao encerrarem o período de internação, nem sempre estão preparados para escolher o que é melhor para seu futuro e conscientes dos desafios que serão enfrentados. Considerando o período desenvolvimental em que se encontram, destaca-se a importância de que os responsáveis por esses jovens, assim como o próprio Estado, na figura dos profissionais da FASE, possam criar circunstâncias mais favoráveis e efetivas para que a adesão a um programa de acompanhamento ocorra de forma mais frequente.

Programas de intervenção descentralizados poderiam ter maior impacto, visto que estariam dentro da própria comunidade onde vivem os jovens, oferecendo uma alternativa às atividades em que estavam habituados a se inserir, tais como o tráfico de drogas. Dessa forma, destaca-se a importância de esforços conjuntos, que envolvam as famílias, os profissionais da área de educação e de saúde e os órgãos responsáveis pela execução das medidas socioeducativas, além de toda a sociedade, no sentido de oferecer alternativas mais adequadas ao desenvolvimento dos jovens. Assim, acredita-se que o acompanhamento aos adolescentes egressos do sistema socioeducativo deveria ser previsto para todos, como um direito do jovem e de sua família, e não como algo opcional.

\section{REFERÊNCIAS}

Abrams, L. S. \& Snyder, S. M. (2010). Youth offender reentry: Models for intervention and directions for future inquiry. Children and Youth Services Review, 32, 1787-1795. doi: 10.1016/j.childyouth.2010.07.023

Agnew, R. \& White, H. R. (1992). An empirical test of general strain theory. Criminology, 30(4), 475-500. doi: 10.1111/j.17459125.1992.tb01113.x

Agnew, R. (2001). Building on the foundation of general strain theory: Specifying the types of strain most likely to lead to crime and delinquency. Journal of Research in Crime and Delinquency, 38(4), 319-361. doi: 10.1177/0022427801038004001

ANCED, Fórum DCA. (2004). Relatório sobre a situação dos direitos da criança e do adolescente no Brasil. Fortaleza, Brasil.

Apel, R. \& Burrow, J. D. (2011). Adolescent victimization and violent self-help. Youth Violence and Juvenile Justice, 9(2), 112133. doi: $10.1177 / 1541204010376939$

Assis, S. G., Avanci, J. Q., Pesce, R. P., \& Ximenes, L. F. (2009). Situação de crianças e adolescentes brasileiros em relação à saúde mental e à violência. Ciência \& Saúde Coletiva, 14(2), 349-361. doi: 10.1590/S1413-81232009000200005

Assis, S. G. \& Souza, E. R. (1999). Criando Caim e Abel - Pensando a prevenção da infração juvenil. Ciência e Saúde Coletiva, 4(1), 131-144.

Collins, R. E. (2004). Onset and desistance in criminal careers: Neurobiology and the age-crime relationship. Journal of Offender Rehabilitation, 39, 1-19. doi: 10.1300/J076v39n03_01

Dell'Aglio, D. D., Koller, S. H., Cerqueira-Santos, E., \& Colaço, V. F. R. (2011). Revisando o Questionário da Juventude Brasileira: Uma nova proposta. In D. D. Dell'Aglio \& S. H. Koller (Eds.). Adolescência e Juventude: vulnerabilidade e contextos de proteção (pp. 259-270). São Paulo: Casa do Psicólogo.

Domburgh, L. V., Loeber, R., Bezemer, D., Stallings, R., \& Stouthamer-Loeber, M. (2009). Childhood predictors of desistance and level of persistence in offending in early onset offenders. Journal of Abnormal Child Psychology, 37, 967-980. doi:10.1007/s10802-009-9329-x

Donnellan, M., Trzesniewski, K., Robins, R., Moffitt, T., \& Caspi, A. (2005). Low self-esteem is related to aggression, antisocial behavior, and delinquency. Psychological Science, 16(4), 328335. doi: 10.1111/j.0956-7976.2005.01535

Formiga, N. S., Aguiar, M., \& Omar, A. (2008). Busca de sensação e condutas anti-sociais e delitivas em jovens. Psicologia Ciência e Profissão, 28(4), 668-681.

Fowler, P. P., Tompsett, C. J., Braciszewski, J. M., Jacques-Tiura, A. J., \& Baltes, B. (2009). Community violence: A meta-analysis on the effect of exposure and mental health outcomes of children and adolescent. Development and Psychopathology, 21, 227259. doi: 10.1017/S0954579409000145

Gallo, A. \& Williams, L. C. A. (2008). A escola como fator de proteção à conduta infracional de adolescentes. Cadernos de Pesquisa, 38(133), 41-59.

Günther, I. A. \& Günther, H. (1998). Brasílias pobres, Brasílias ricas: perspectivas de futuro entre adolescentes. Psicologia: Reflexão e Crítica, 11, 191-207. doi: 10.1590/S0102-79721998000200003

Hein, A. (2004). Factores de riesgo y delincuencia juvenil: Revisión de la literatura nacional e internacional. Fundación Paz e Ciudadana. Retrieved in Sept 04, 2010, from http:/www. pazciudadana.cl/docs/pub_20090623190509.pdf

Higgins, G. E., Jennings, W. G., Marcumc, C. D., Ricketts, M. L., \& Mahoney, M. (2011). Developmental trajectories of nonsocial reinforcement and offending in adolescence and 
young adulthood: An exploratory study of an understudied part of social learning theory. Journal of Criminal Justice, 39, 60-66. doi: 10.1016/j.jcrimjus.2010.10.005

Horney, J. D., Osgood, D. W., \& Marshall, I. H. (1995). Criminal careers in the short-term: Intraindividual variability in crime and its relation to local life circumstances. American Sociological Review, 60(5), 655-673.

Hutz, C. S. \& Zanon, C. (2011). Revisão da adaptação, validação e normatização da escala de autoestima de Rosenberg. Avaliação Psicológica, 10(1), 41-49.

Iselin, A.M.R., Mulvey, E. P., Loughran, T. A., Chung, H. L., \& Schubert, C. A. (2012). A longitudinal examination of serious adolescent offenders' perceptions of chances for success and engagement in behaviors accomplishing goals. Journal of Abnormal Child Psychology, 40, 237-249. doi: 10.1007/s10802011-9561-z

Kesller, F., Diemen, L., Seganfredo, A. C., Brandão, I., Saibro, P., Scheidt, B., Grillo, R., \& Ramos, S. (2003). A dinâmica do adolescente envolvido com drogas. Revista de Psiquiatria do Rio Grande do Sul, 25(SUPL I), 33-41.

Kirkcaldy, B. D., Siefen, G., Surall, D., \& Bischoff, R. J. (2004). Predictors of drug and alcohol abuse among children and adolescents. Personality and Individual Differences, 36, 247265. doi: 10.1016/S0191-8869(03)00082-5

Lee, Z., Klaver, J. R., Hart, S. D., Moretti, M. M., \& Douglas, K. S. (2009). Short-term stability of psychopathic traits in adolescent offenders. Journal of Clinical Child \& Adolescent Psychology, 38(5), 595-605. doi: 10.1080/15374410903103536

Maldonado-Molina, M. M., Piquero, A. R., Jennings, W. G., Bird, H., \& Canino, G. (2009). Trajectories of delinquency among Puerto Rican children and adolescents at two sites. Journal of Research in Crime and Delinquency, 46, 144-182. doi: $10.1177 / 0022427808330866$

Mason, W.A. \& Windle, M. (2002). Reciprocal relations between adolescent substance use and delinquency: a longitudinal latent variable analysis. Journal of Abnormal Psychology, 111, 63-76. doi: 10.1037/0021-843X.111.1.63

Mennis, J. \& Harris, P. (2011). Contagion and repeat offending among urban juvenile delinquents. Journal of Adolescence, 34 , 951-963. doi: 10.1016/j.adolescence.2010.12.001

Moffitt, T. E. (1993). Adolescence-limited and life-course-persistent antisocial behavior: A developmental taxonomy. Psychological Review, 100(4), 674-701.

Moffitt, T. E. (2006). A review of research on the taxonomy of life-course persistent versus adolescence-limited antisocial behavior. In F. T. Cullen, J. P. Wright, \& K. R. Blevins (Eds.). Taking stock: The status of criminological theory - Advances in criminological theory (pp. 277-311). New Brunswick, New Jersey: Transaction Publishers.

Nijhof, K. S., Vermulst, A., Scholte, R. H. J., Dam, C. V., Veerman, J. W., \& Engels, R. C. M. E. (2011). Psychopathic traits of Dutch adolescents in residential care: Identifying subgroups. Journal of Abnormal Child Psychology, 39, 59-70. doi: 10.1007/s10802010-9445-7.

Pacheco, J., Alvarenga, P., Reppold, C., Piccinini, C. A., \& Hutz, C. S. (2005). Estabilidade do comportamento anti-social na transição da infância para a adolescência: Uma perspectiva desenvolvimentista. Psicologia: Reflexão e Crítica, 18(1), 55-61. doi: 10.1590/S0102-79722005000100008
Park, N. S., Lee, B. S., Sun, F., Vazsonyi, A. T., \& Bolland, J. M. (2010). Pathways and predictors of antisocial behaviors in African American adolescents from poor neighborhoods. Children and Youth Services Review, 32(3), 409-415. doi: 10.1016/j.childyouth.2009.10.012

Paton, J., Crouch, W., \& Camic, P. (2009). Young offenders' experiences of traumatic life events: A qualitative investigation. Clinical Child Psychology Psychiatry, 14(1), 43-62. doi: 10.1177/1359104508100135

Pereira, S. E. F. N. \& Sudbrack, M. F. O. (2008). Drogadição e atos infracionais na voz do adolescente em conflito com a lei. Psicologia: Teoria e Pesquisa, 24(2), 151-159. doi: 10.1590/ S0102-37722008000200004

Rosenberg, M. (1989). Society and the adolescent self-image. Princeton, NJ: Princeton University Press.

Sampson, R. J. \& Laub, J. H. (2003). Life-course desisters: Trajectories of crime among delinquent boys followed to age 70 . Criminology, 41(3), 555-592. doi: 10.1111/j.1745-9125.2003. tb00997.x

Silva, D. F. M. (2002). O desenvolvimento das trajetórias do comportamento delinqüente em adolescentes infratores. Unpublished doctoral dissertation. Programa de Pós-Graduação em Psicologia. Porto Alegre: Universidade Federal do Rio Grande do Sul.

Trzesniewski, K., Donnellan, M., \& Robins, R. (2003). Stability of self-esteem across the life span. Journal of Personality and Social Psychology, 84(1), 205-220. doi: 10.1037/00223514.84.1.205

Wainer, F. W. (2006). Prevención social del delito: Pautas para una intervención temprana en niños y jóvenes. Fundación Hanns Seidel Stiftung \& Fundación Paz e Ciudadana. Retrieved in Aug 28, 2010, from http://www.pazciudadana.cl/docs/ pub_20090618132605.pdf

Waiselfisz, J. J. (2013). Mapa da violência-homicídios e juventude no Brasil. Brasília: Secretaria Geral da Presidência da República.

Warr, M. (1998). Life-course transitions and desistance from crime. Criminology, 36(2), 183-216. doi: 10.1111/j.1745-9125.1998. tb01246.x

Zapata, F. B. (2010). Internação: Medida socioeducativa? Reflexões sobre a socioeducação associada à privação de liberdade. Revista da Defensoria Pública: Direito da Criança e do Adolescente, (ed. esp.), 43-50. Retrieved in Sept 10, 2012, from http://www. defensoria.sp.gov.br/dpesp/repositorio/0/Edepe_Revista.pdf

Zappe, J. G. \& Dias, A. C. G. (2012). Adolescência, violência e uso de drogas: Um estudo de casos múltiplos. Adolescência \& Saúde, 9(2), 30-36.

\section{Autores:}

Fernanda Ludke Nardi - Doutora em Psicologia e Psicóloga da Prefeitura Municipal de Porto Alegre.

Débora Dalbosco Dell'Aglio - Doutora em Psicologia e Docente do Programa de Pós-Graduação em Psicologia, Universidade Federal do Rio Grande do Sul.

\section{Endereço para correspondência:}

Fernanda Ludke Nardi

Rua Dr. Mário Totta, 1301 - Tristeza

CEP 91920-130 Porto Alegre, RS, Brasil

E-mail: fernanda.nardi@yahoo.com.br

Recebido em: 10/01/2013

Aceito em: 24/06/2014 